\title{
Orientation Tuning Depends on Spatial Frequency in Mouse Visual Cortex
}

\author{
(D) Inbal Ayzenshtat, Jesse Jackson, and Rafael Yuste
}

DOI:http://dx.doi.org/10.1523/ENEURO.0217-16.2016

NeuroTechnology Center, Department of Biological Sciences, Columbia University, New York, New York 10027

\begin{abstract}
The response properties of neurons to sensory stimuli have been used to identify their receptive fields and to functionally map sensory systems. In primary visual cortex, most neurons are selective to a particular orientation and spatial frequency of the visual stimulus. Using two-photon calcium imaging of neuronal populations from the primary visual cortex of mice, we have characterized the response properties of neurons to various orientations and spatial frequencies. Surprisingly, we found that the orientation selectivity of neurons actually depends on the spatial frequency of the stimulus. This dependence can be easily explained if one assumed spatially asymmetric Gabor-type receptive fields. We propose that receptive fields of neurons in layer 2/3 of visual cortex are indeed spatially asymmetric, and that this asymmetry could be used effectively by the visual system to encode natural scenes.
\end{abstract}

Key words: visual cortex; cortical tuning; orientation selectivity; spatial frequency; two-photon; calcium imaging; mouse; asymmetry; Gabor model

\section{Significance Statement}

In this manuscript, we show that the orientation selectivity of neurons in primary visual cortex of the mouse is highly dependent on the stimulus spatial frequency (SF). This dependence is demonstrated quantitatively by a decrease in the selectivity strength of cells in nonoptimum SF, and, more importantly, it is also demonstrated qualitatively by a shift in the preferred orientation of cells in nonoptimum SF. We show that a receptive-field model of a $2 \mathrm{D}$ asymmetric Gabor, rather than a symmetric one, can explain this surprising observation. Therefore, we propose that the receptive fields of neurons in layer $2 / 3$ of mouse visual cortex are spatially asymmetric, and this asymmetry could be used effectively by the visual system to encode natural scenes.

\section{Introduction}

Neurons in the primary visual cortex (V1) have been traditionally described by their receptive field (RF) struc-

Received July 28, 2016; accepted September 14, 2016; First published September 21, 2016.

The authors declare no competing financial interests.

Author contributions: I.A. designed research; I.A. and J.J. performed research; I.A. analyzed data; I.A. and R.Y. wrote the paper.

This study was supported by a Marie Curie International Outgoing Fellowship (to I.A.), a Canadian Institute for Health Research grant (to J.J.), National Eye Institute Grants DP1EY024503 and R01EY011787, and Defense Advanced Research Projects Agency SIMPLEX Grant N66001-15-C-4032. This material is based upon work supported by, or in part by, the U.S. Army Research Laboratory and the U.S. Army Research Office under contract number W911NF-12-1-0594 (MURI). ture and their response characteristics (Hubel and Wiesel, 1962; Skottun et al., 1991). They are classified into two major groups, simple and complex, and exhibit spatially localized receptive fields that consist of distinct elongated

\footnotetext{
Acknowledgments: We thank Darcy Peterka, Julia Sable, and Yeonsook Shin for technical support; and Guy Zurawel for fruitful discussions.

Correspondence should be addressed to Inbal Ayzenshtat, Department of Biological Sciences, NeuroTechnology Center, Columbia University, New York, NY 10027. E-mail: inbalayzen@gmail.com.

DOI:http://dx.doi.org/10.1523/ENEURO.0217-16.2016

Copyright (C) 2016 Ayzenshtat et al.

This is an open-access article distributed under the terms of the Creative Commons Attribution 4.0 International, which permits unrestricted use, distribution and reproduction in any medium provided that the original work is properly attributed.
} 
ON and OFF subfields. In mouse visual cortex, $\sim 75 \%$ of the orientation-selective neurons in layer $2 / 3$ are classified as simple cells, showing response characteristics similar to simple cells in visual cortex of monkeys or cats (Niell and Stryker, 2008).

To capture the selectivity of a neuron to a certain feature of a visual stimulus (e.g., orientation, spatial frequency (SF), size, position, and speed), it is convenient to measure one-dimensional (1D) tuning curves that show the average response of a neuron to a specific feature value. Customary tuning models propose that the response strength of a neuron can be predicted based on the similarity between the optimal stimulus of a neuron and the given stimulus. From the 1D tuning curves, which describe the response behavior to one stimulus feature, one can calculate several parameters such as the preferred stimulus, the strength of the selectivity, or the width of the selectivity that quantifies the specificity level of the neuron. In visual cortex, for example, since neurons are highly responsive to lines and edges, such curves, which commonly characterize simple cells in $\mathrm{V} 1$, are the orientation-tuning and spatial frequency-tuning curves. However, reducing the complexity of the RF spatial structure to 1D tuning curves in order to study individual features, comes at a cost of losing information that might be critical for understanding the neuronal representation of sensory information. Here, we measured the population responses of neurons in L2/3 of mice V1 to drifting gratings that varied in both orientation and SF. We used in vivo twophoton $\mathrm{Ca}^{2+}$ imaging to measure evoked responses from hundreds of V1 neurons. Accordingly, we calculated a two-dimensional (2D) tuning matrix, and studied the relationship between orientation and SF selectivity. Then, we compared orientation-tuning curves in various SFs. First, we found that the orientation selectivity of a neuron depends strongly on the stimulus SF, such that when we presented gratings with higher or lower SFs than the optimum, the orientation selectivity was significantly reduced. In addition to a quantitative change in the selectivity strength of the neurons, we also observed a qualitative change in the preferred stimulus of the neuron. As we moved away from the optimal SF, to either lower or higher SFs, there was a significant shift in the preferred orientation (Pref) of the neurons. Dependence between orientation and SF selectivity of cells has been previously suggested in the visual cortex of primates and cats (Andrews and Pollen, 1979; Vidyasagar and Sigüenza, 1985; Webster and De Valois, 1985; Jones et al., 1987; Zhu et al., 2010).

In order to explain this dependence between orientation selectivity and SF, we used the common Gabor model (Gabor, 1946) to predict the neuronal response to various stimuli. A Gabor filter is a Gaussian modulated sinusoid, which well describes the receptive fields of simple cells and successfully models their responses (Marcelja, 1980; Field and Tolhurst, 1986; Jones and Palmer, 1987b). However, the classic Gabor model, even though it succeeds in predicting multiple neuronal responses, cannot capture the full variety and complexity of the visual system. And, indeed, we found that the classic mathematical descrip- tion of a 2D symmetric Gabor model (with either odd or even amplitude symmetry) cannot account for our experimental findings. However, spatially modifying the classic model to introduce a 2D asymmetry by way of tilting the Gabor against its elongated axis generates a fundamental change in the response predictions, which qualitatively explains our experimental observations.

The modified Gabor model presented in this article can explain the response characteristics of a population of neurons and suggests that the receptive field of many cells in layer $2 / 3$ of visual cortex of mice demonstrates a central asymmetry in its 2D spatial organization.

\section{Materials and Methods}

\section{Animals}

Animal handling and experimentation were performed in accordance with the National Institutes of Health and Columbia University institutional animal care guidelines. Animals of both sexes were used and were housed in a temperature-controlled environment on a $12 \mathrm{~h}$ light/dark cycle. We used a total of five mice, either WT or VIP-Cre crossed with LSL-tdTomato [postnatal day 40 (P40) to P80; The Jackson Laboratory].

\section{Surgery}

The mice were placed on a warming plate $\left(37^{\circ} \mathrm{C}\right)$ and anesthetized with isoflurane (initially $2 \%$, and reduced to $1-1.5 \%$ during surgery) administered via nose cone. A custom-made titanium head plate was attached to the skull using dental cement. Subsequently, a craniotomy $(\sim 1 \times 1 \mathrm{~mm})$ was made over the primary visual cortex (3.5-4.5 mm posterior to bregma, 2.3-2.7 $\mathrm{mm}$ lateral to midline; putative monocular region) using a dental drill (Osada). An ophthalmic ointment was applied on both eyes to protect the eyes from dehydration during surgery, and was removed during visual stimulation.

\section{Dye loading}

For bulk loading of cortical neurons, Oregon Green Bapta-1 (OGB-1) AM (Molecular Probes) was first mixed with $4 \mu \mathrm{l}$ of pluronic acid (20\% in DMSO) and further diluted in $35 \mu \mathrm{l}$ of dye buffer $(150 \mathrm{~mm} \mathrm{NaCl}, 2.5 \mathrm{~mm} \mathrm{KCl}$, and $10 \mathrm{~mm}$ HEPES, pH 7.4). Sulforhodamine-101 (SR101; Molecular Probes) at a concentration of $50 \mu \mathrm{m}$ was added to the solution to label astrocytes (Nimmerjahn et al., 2004). Animals were head fixed, and the dye was slowly pressure injected into the left visual cortex at a depth of $\sim 130-200 \mu \mathrm{m}$ below the dura surface (layer 2/3) at an angle of $30^{\circ}$ through a patch pipette (outer diameter, $\sim 1-2 \mu \mathrm{m})$ using a Picospritzer II. Two to four injections were carried out at $10 \mathrm{psi}$ for $8 \mathrm{~min}$, each under visual control of a two-photon imaging microscope [850 nm excitation; $10 \times$ water-immersion objective; 0.5 numerical aperture (NA); Olympus]. After dye injections, the exposed cortex was covered with agarose (1.5-2\%; Sigma-Aldrich) and a cover glass (World Precision Instruments) to reduce brain motion. Data collection began 60-90 min after injections to ensure dye uptake across a large number of cells. During data collection, light anesthesia was maintained by isoflurane $(0.8-0.9 \%)$ administered via nose 
cone (KOPF Instruments). Heart rate, respiration, and oxygen saturation were monitored throughout the experiments using MouseOx (STARR Life Sciences Corp), and the respiration rate was used to monitor and control anesthesia levels.

\section{Two-photon $\mathrm{Ca}^{2+}$ imaging}

Imaging was performed with a two-photon moveable objective microscope (Sutter Instrument) and a modelocked dispersion-precompensated Ti:sapphire laser (Chameleon Vision II, Coherent). Frames were scanned through a $20 \times(0.95 \mathrm{NA}$; Olympus) or $25 \times(1.05 \mathrm{NA}$, Olympus) water immersion objective. Laser intensity was controlled via Pockels cell (Conoptics) and ranged between 20 and $70 \mathrm{~mW}$. Scanning and image acquisition were controlled using Mscan (4.07 frames/s for $512 \times 512$ pixels; Sutter Instrument). OGB-1 fluorescence was excited at $950 \mathrm{~nm}$. Fluorescence changes collected with a $20 \times$ objective typically varied between $5 \%$ and $50 \%$, and between $10 \%$ and $70 \%$ with a $25 \times$ objective. Emission was collected using green $(535 / 50 \mathrm{~nm})$ and red $(610 / 75$ $\mathrm{nm}$ ) filters (Chroma) simultaneously on two photomultiplier tubes.

\section{Visual stimulation}

Visual stimuli were generated in MATLAB (RRID: SCR_001622) using Psychophysics toolbox (Brainard, 1997; Pelli, 1997; Kleiner et al., 2007) and displayed on a gamma-corrected LCD monitor (Dell; 19 inches, $60 \mathrm{~Hz}$ refresh rate) positioned $15 \mathrm{~cm}$ from the contralateral eye, at $\sim 45^{\circ}$ to the long axis of the animal (spanning $\sim 114^{\circ}$ vertical by $\sim 140^{\circ}$ horizontal of visual space). The presentation of visual stimuli was synchronized with image acquisition using Mscan (Sutter Instrument) and a routine written in MATLAB, such that each stimulus presentation was triggered on the beginning of frame acquisition. The actual time of stimulus presentation was detected with a silicon photodiode (Hamamatsu) attached to the bottom right corner of the screen.

We presented square-wave drifting gratings (100\% contrast) for $670 \mathrm{~ms}$, followed by $3-5 \mathrm{~s}$ of uniform gray background (the mean luminance of the gratings) plus a blank condition. The gratings orientation was perpendicular to the drift direction. Gratings were presented at 12 directions of motion (in $30^{\circ}$ steps), three sets of four or five spatial frequencies: $[0.02,0.03,0.04,0.06$ cycles per degree (cpd)], [0.01 $0.020 .040 .06 \mathrm{cpd}$ ], or [0.01 0.020 .04 $0.080 .16 \mathrm{cpd}$ ], and a temporal frequency of $1.5 \mathrm{~Hz}$. All stimuli were block randomized and repeated 5-10 times, and the initial phase of the drifting gratings was kept constant across all trials. Stimuli were presented in a pseudorandom order, but time courses are shown after sorting (Fig. 1C).

\section{Data analysis}

Image analysis

All data analyses were performed using built-in and custom software written in MATLAB (MathWorks). Images were first converted to TIFF format and registered to correct for $x-y$ motion using Turboreg plug-in in ImageJ (Thévenaz et al., 1998; RRID:SCR_014308). Regions of interest (ROls) were drawn around each cell using a semiautomated algorithm based on fluorescence intensity (mean projection), florescence change (SD projections), and cell size and shape, and were adjusted by visual inspection. Glia cells were excluded from further analysis using SR101 staining, which selectively labels astrocytes in rodent neocortex (Nimmerjahn et al., 2004). Pixels were averaged within each ROI for each image frame. Baseline $\mathrm{Ca}^{2+}$ fluorescence was computed for each trial as the mean over $2 \mathrm{~s}$ prestimulus. Then, fluorescence values were converted to the percentage change above baseline [response amplitude $(\Delta F / F)$ ] according to the following: $\Delta F / F=\left(F_{1}-F\right) / F$, where $F$ is the baseline fluorescence and $F_{1}$ is the instantaneous fluorescence signal averaged over two frames $(\sim 500 \mathrm{~ms})$ following stimulus onset $\left(F_{0+2}\right.$, $F_{0+3}$, where $F_{0}$ is the stimulus onset frame).

Responsiveness and reliability criteria were defined as previously described (Marshel et al., 2011). Briefly, neurons were considered responsive if their mean $\Delta F / F$ to any stimulus exceeds $6 \%$. Reliability $(\delta)$ was determined according to the following:

$$
\delta=\frac{\mu_{\max }-\mu_{\text {blank }}}{\sigma_{\max }-\sigma_{\text {blank }}},
$$

where $\mu_{\max }$ and $\sigma_{\max }$ are the mean and SD of the response to the preferred stimulus, respectively, and $\mu_{\text {blank }}$ and $\sigma_{\text {blank }}$ are the mean and SD of the response to the blank stimulus, respectively. Neurons were considered reliable if $\delta>1$. Only cells that demonstrated visual responsiveness and reliability were chosen for further analysis, which excluded between $38 \%$ and $43 \%$ of the total number of cells we observed per field of view (FOV). Therefore, we analyzed $85.4 \pm 3.6$ reliably responsive cells of $156.2 \pm 7.67$ cells in total (mean \pm SEM; $n=5$ mice).

\section{Visual tuning}

To calculate tuning curves, we averaged the evoked responses $(\Delta F / F)$ over two frames following stimulus presentation. Then we averaged the response over the number of repetitions (5-10) per stimulus direction (12 directions). Direction tuning curves generated from OGB-1 fluorescence are comparable to those recorded with electrophysiological techniques (Kerlin et al., 2010; Marshel et al., 2011).

Orientation selectivity index (OSI) was computed as follows:

$$
\text { OSI }=\frac{\mu_{\max }-\mu_{\text {orth }}}{\mu_{\max }+\mu_{\text {orth }}}
$$

where $\mu_{\max }$ is the mean response to the Pref and $\mu_{\text {orth }}$ is the mean response to the orthogonal orientation (Orth; average of both directions). Only cells that demonstrated an $\mathrm{OSI} \geq 0.3$ were chosen for tuning comparisons.

Orientation-tuning curves were fitted with the sum of two Gaussians of identical width, as follows: 
A

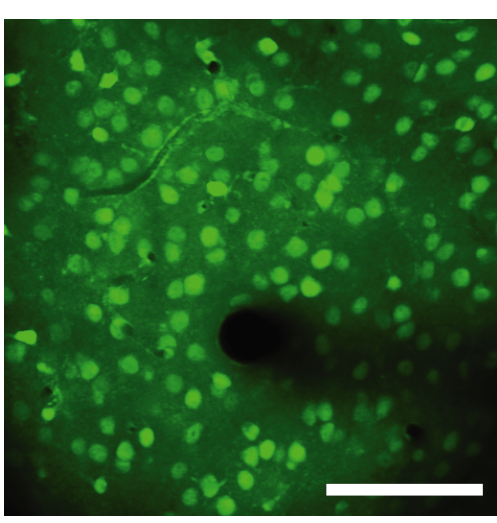

C

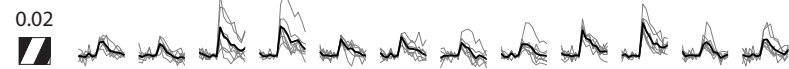

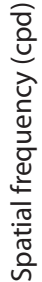

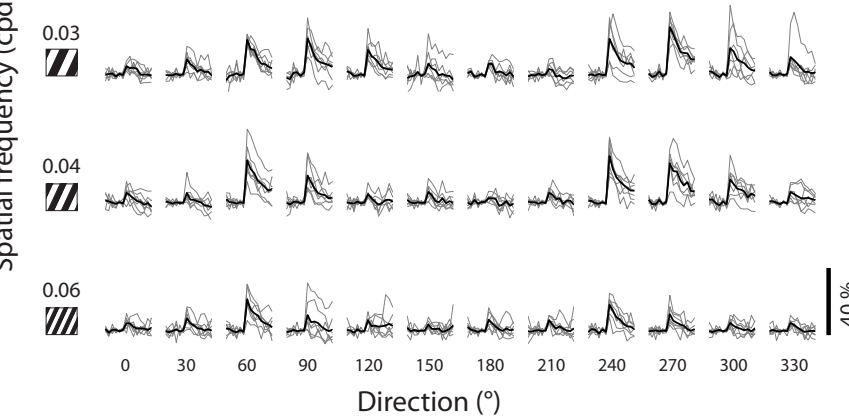

D
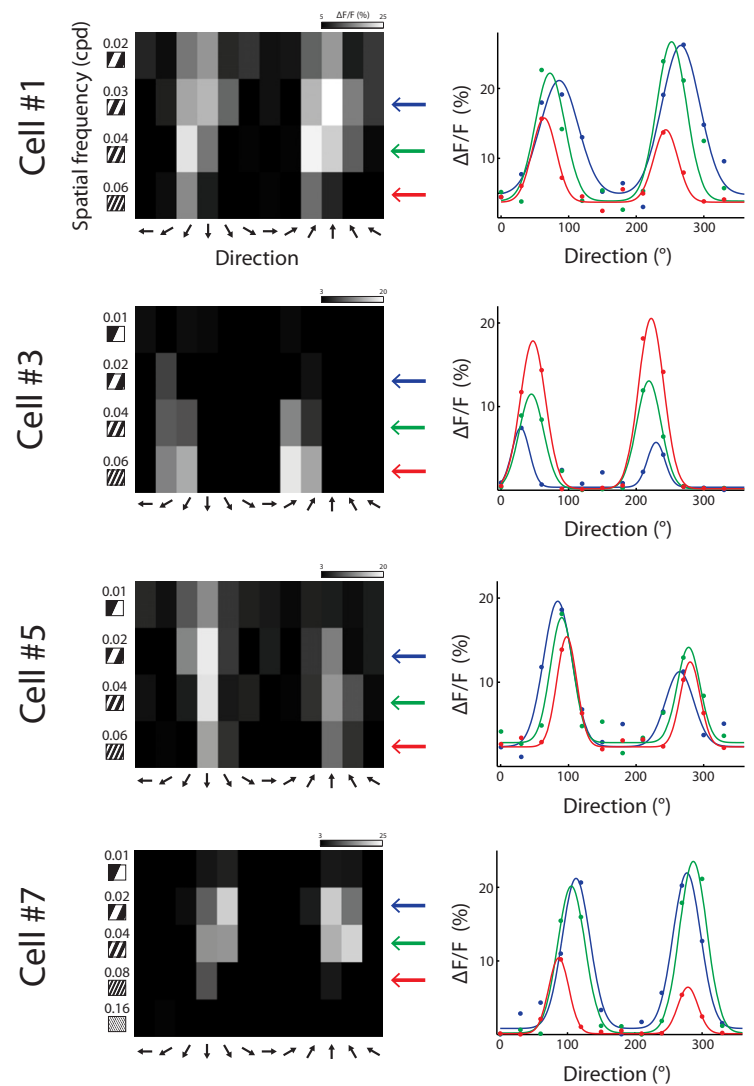

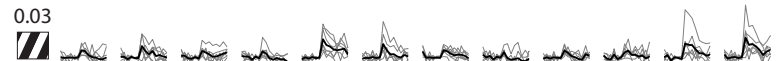

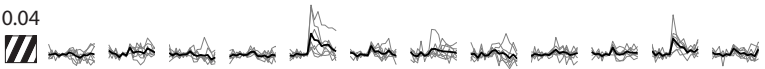

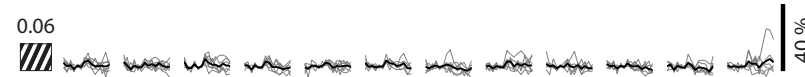
Direction $\left(^{\circ}\right)$
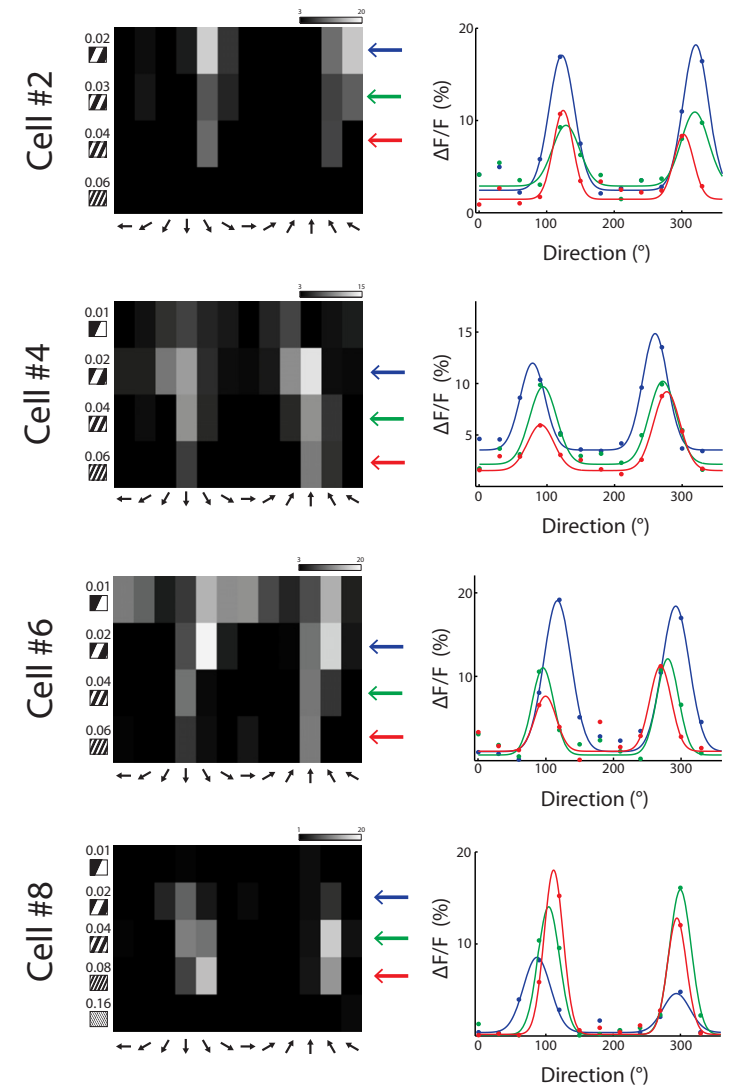

eNeuro.org

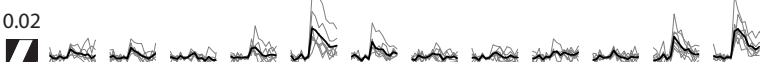
$\begin{array}{llllllllllll}0 & 30 & 60 & 90 & 120 & 150 & 180 & 210 & 240 & 270 & 300 & 330\end{array}$ $\stackrel{Ð}{\bar{\Xi}}$ 
Figure 1. In vivo imaging of visual-evoked responses of layer $2 / 3$ neurons. $\boldsymbol{A}$, Top, A two-photon image (maximum projection) from L2/3 neurons in mouse primary visual cortex, loaded with OGB-1. Scale bar, $100 \mu \mathrm{m}$. B, Example traces of $\mathrm{Ca}^{2+}$ signals from 10 cells during the presentation of drifting gratings with 4 different spatial frequencies and 12 directions. $\boldsymbol{C}$, $\mathrm{Ca}^{2+}$ responses of two neurons, displayed as a matrix of all stimulus conditions. Columns indicate the direction of motion of the gratings, and rows indicate their SF. Each trial is shown in gray $(n=7)$; average response across trials of a given stimulus is shown in black. $\boldsymbol{D}$, Tuning matrix of eight cells (cells 1 and 2 are shown in $\boldsymbol{C}$ ). Left, Response matrices evoked by each stimulus. Pixels intensity corresponds to the average $\Delta F / F$ over two frames poststimulus presentation and over seven repetitions. Right, Direction-tuning curves fitted with a double Gaussian, measured in various SFs. Colors correspond to SFs marked with arrows next to the matrices on the left.

$$
R(\theta)=\alpha_{0}+\alpha_{1} e^{\frac{-\left(\theta-\theta_{0}\right)^{2}}{2 \sigma^{2}}}+\alpha_{2} e^{\frac{-\left(\theta-\theta_{1}\right)^{2}}{2 \sigma^{2}}},
$$

where $R(\theta)$ is the averaged response to gratings with direction $\theta$, and $\alpha_{0}$ is the mean $\Delta F / F$ of the four lowest points in the curve. $\alpha_{1}$ and $\alpha_{2}$ are the amplitudes of the two Gaussians, $\theta_{0}$ is the preferred direction, $\theta_{1}$ is the null direction, and $\sigma$ is the SD of the Gaussian function. The sum of two Gaussians fitting was constrained, according to Mazurek et al. (2014), by using several initial conditions, considering the fit with the lowest least square error as the best fit of the data. Peak responses were the maximum $\Delta F / F$ values of the 2D Gaussian fit curve. Half-width at half-height $(\mathrm{HWHH})$ was computed as follows:

$$
H W H H=\sqrt{2 \ln 2} \sigma
$$

where $\sigma$ is the SD of the Gaussian function.

To further assess the statistical robustness of the tuning curve fitting, we applied a bootstrap method where we randomly resampled the data with replacement for each cell and obtained a distribution of response values. This procedure was repeated 100 times, where each repetition was fitted with a double Gaussian (Mazurek et al., 2014). This yielded a distribution of values for each of the tuning curve parameters. The mean values of the resampled data were then used for comparing population parameters showing the same statistical significance as achieved by comparing parameters of tuning curves obtained by fitting the data that included all the trials.

Additionally, orientation selectivity was assessed using a metric based on 1 - circular variance (CirVar; Ringach et al., 2002), as follows:

$$
1-\operatorname{CirVar}=\frac{\sum R(\theta) \exp (2 i \theta)}{\sum R(\theta)}
$$

where $R(\theta)$ is the averaged response to gratings with orientation $\theta$. This metric takes into account both tuning width and depth of modulation, and was found to be more reliable than extracting parameters following curve fitting, and more sensitive for detecting differences in selectivity between two populations (Piscopo et al., 2013; Mazurek et al., 2014).

\section{D Gabor model}

The two-dimensional Gabor function is the product of a 2D sinusoid wave with a circular Gaussian envelope (see Fig. 3A), which was defined as follows:

$$
g(x, y)=\exp \left(-\frac{x^{2}+\gamma^{2} y^{\prime 2}}{2 \sigma^{2}}\right) \cos \left(2 \pi \frac{x^{\prime}}{\lambda}+\psi\right)
$$

$$
\begin{aligned}
& x^{\prime}=x \cos \theta+y \sin \theta, \\
& y^{\prime}=-x \sin \theta+y \cos \theta,
\end{aligned}
$$

where $\sigma$ is the SD of the Gaussian envelope, which determines the size of the receptive field; $\gamma$ is the spatial aspect ratio of the Gaussian, which determines the ellipticity of the Gabor (in a circular Gaussian $\gamma=1$ ); $\lambda$ is the wavelength of the sinusoid; $\theta$ is the orientation; and $\psi$ is the phase offset.

In the tilted Gabor model, we introduced another parameter, which is the angle of the Gaussian tilt $(\phi)$ with respect to the sinusoid wave. To generate a tilted Gabor filter, we first generated a symmetric 2D Gaussian with a spatial aspect ratio of $\gamma=0.5$ (see also Fig. 4B) and then applied a tilt by multiplying it with a $2 \mathrm{D}$ rotation matrix $A$ with $\phi=30^{\circ}$, as follows:

$$
A=\left[\begin{array}{cc}
\cos \phi & -\sin \phi \\
\sin \phi & \cos \phi
\end{array}\right]
$$

\section{Calculating the predicted response}

To calculate the predicted response, $R(I)$, of a neuron, we computed the inner product between the RF $f(x, y)$ either a symmetric or a tilted Gabor filter, and the stimulus $I(x, y)$ - square-wave gratings drifting in 180 different orientations with $1^{\circ}$ interval (see Fig. 3B, examples). Since we measured neuronal responses by recording $\mathrm{Ca}^{2+}$ signals, we obtained tuning matrices by maximizing the averaged evoked response per stimulus. Therefore, to compute the predicted response, we calculated the inner product per stimulus orientation for each phase of the stimulus between 0 and $2 \pi$ (21 samples, $\pi / 10$ apart; see Fig. 5) and then maximized the response across phase.

\section{Results}

\section{Layer 2/3 cells in mouse $\mathbf{V} 1$ show dependence of orientation selectivity on spatial frequency}

In lightly anesthetized mice, we identified cells in layer $2 / 3$ of visual cortex using two-photon $\mathrm{Ca}^{2+}$ imaging and monitored the activity of neuronal populations (Marshel et al., 2011; Miller et al., 2014) evoked by a brief visual stimulus presented to the contralateral visual field. We characterized the response dynamics by optically recording $\mathrm{Ca}^{2+}$ signals of OGB-1 from cells in upper layer 2/3 (depth, $\sim 130-200 \mu \mathrm{m} ; n=5$ animals) in a typical FOV (Fig. 1A,B). Specifically, we measured spatial frequencytuning and direction-tuning curves of each neuron in our FOV by averaging across trials the response to each stimulus (Fig. 1C,D). Stimuli were square-wave drifting gratings presented at $1.5 \mathrm{~Hz}$ for $670 \mathrm{~ms}$ (approximately one cycle) and varied across 12 directions and four or five SFs (see Materials and Methods). 
A

Pref response $(\% \Delta \mathrm{F} / \mathrm{F})$

Orth response $(\% \Delta F / F)$
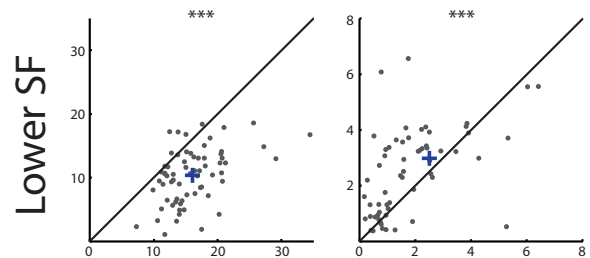

B

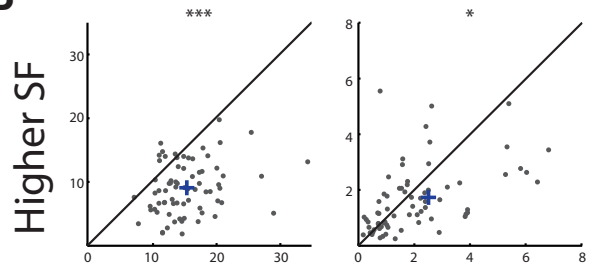

C

$\Delta$ Preferred Orientation $\left({ }^{\circ}\right) \Delta$ Preferred Orientation $\left({ }^{\circ}\right)$

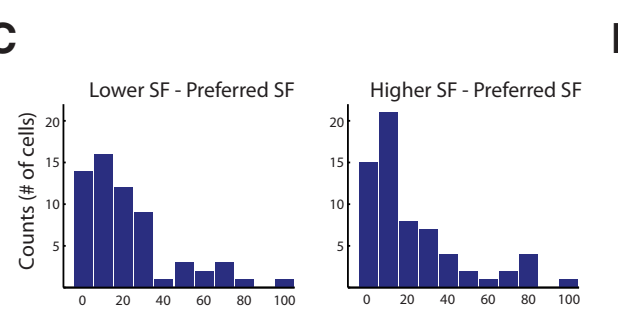

OSI

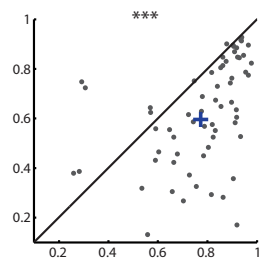

1 - CirVar

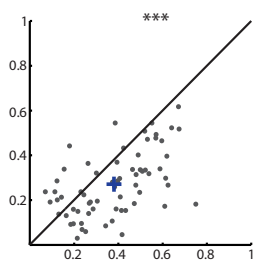

Preferred SF
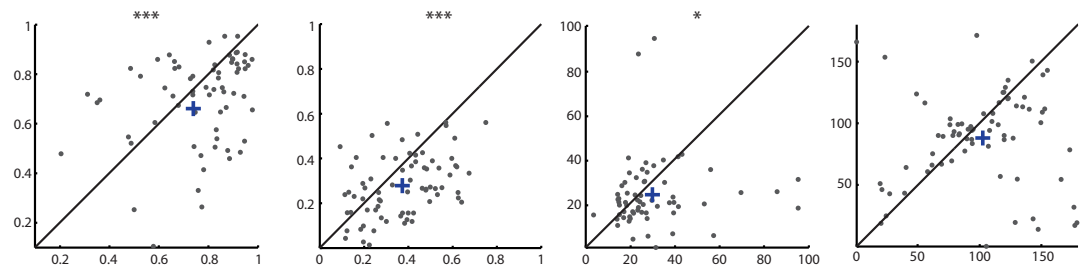

Preferred SF
HWHH $\left({ }^{\circ}\right)$

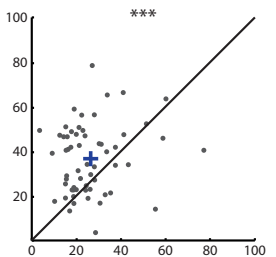

Preferred Orientation $\left(^{\circ}\right)$

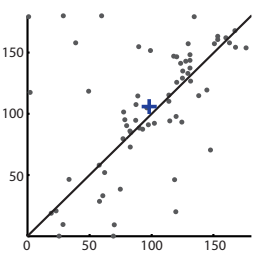

D

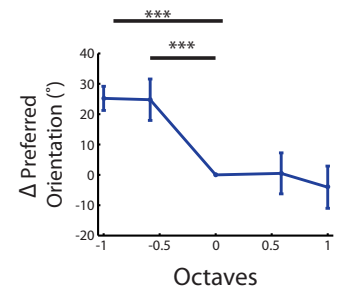

E
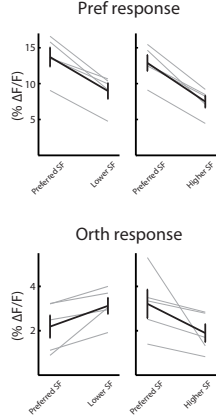
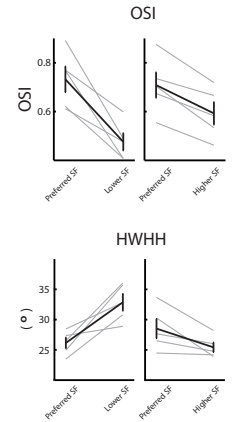

\section{(n)}


A
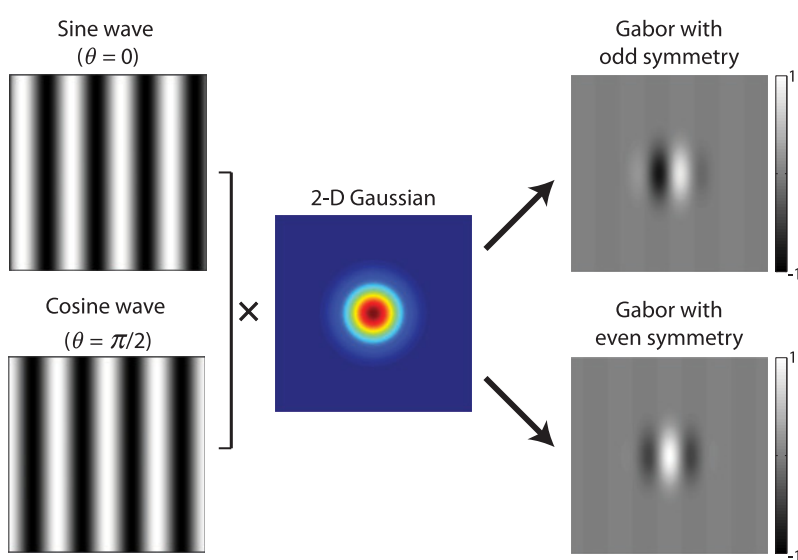

C
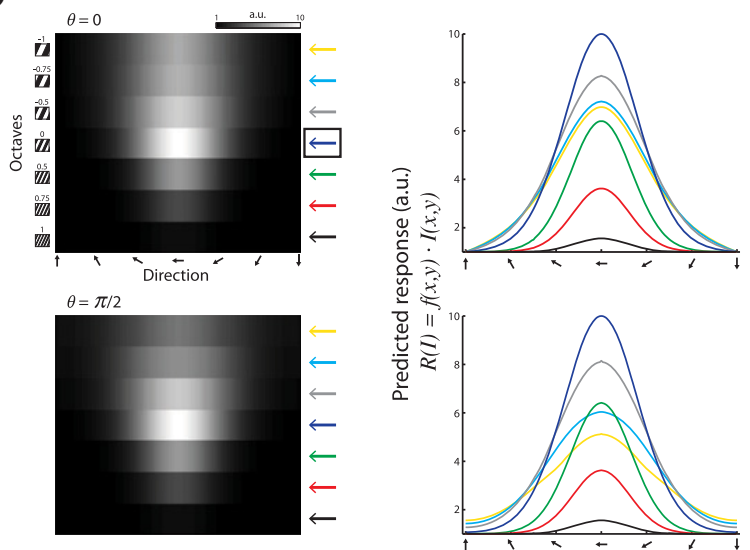

B

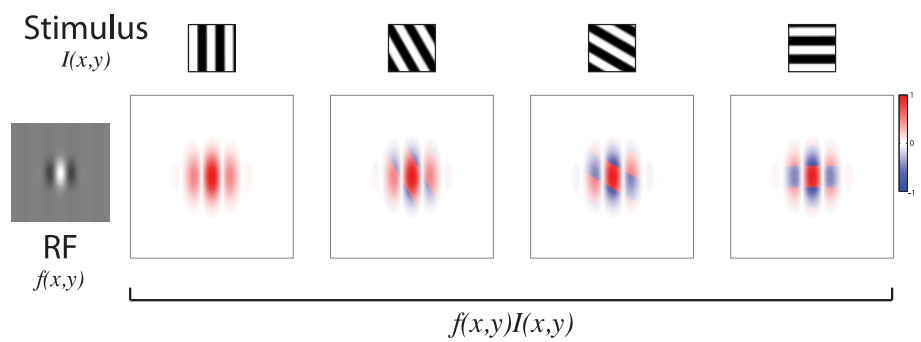

D
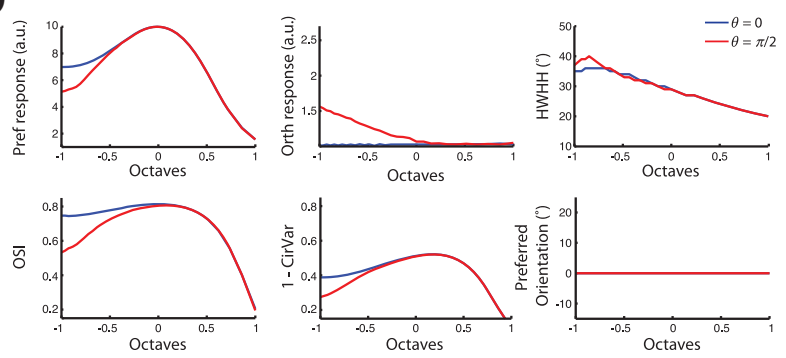

Figure 3. Predicting the responses of a simple cell based on an RF model of a 2D symmetric Gabor model. A, A 2D-oriented Gabor function: a sinusoidal plane wave weighted by a Gaussian envelope in two different phases: $\theta=0$ (sine wave) or $\theta=\pi / 2$ (cosine wave) shown at the top and bottom panels that generate Gabor filters with odd and even symmetry, respectively. $\boldsymbol{B}$, Constructing spatiotemporally oriented impulse responses from gratings stimuli drifting against a Gabor RF. Top, Examples of stimuli with four different orientations. Bottom, Left, An example of a Gabor RF with $\theta=\pi / 2$. Bottom, Right, Four examples where each box depicts the overlap of the grating stimulus crossing the RF in a phase that yields the maximum response, calculated as the inner product between the stimulus and the RF. $\boldsymbol{C}$, A predicted response tuning matrix computed as the inner product between the Gabor RF model shown in $\boldsymbol{A}$, and stimuli of square-wave gratings with various orientations ( $1^{\circ}$ interval) and seven SFs. Each pixel represents the inner product between the RF and the stimulus (maximized across phase, see Materials and Methods). The row marked with a blue arrow on the right denotes the preferred SF and was taken as a reference for comparing other SFs. Right, Orientation tuning curves at various SFs, color coded according to the arrows shown next to the predicted tuning matrix. Top and bottom panels correspond to RF with $\theta=0$ and $\theta=\pi / 2$, respectively. a.u., Arbitrary units. $\boldsymbol{D}$, Comparison of orientation tuning parameters between various SFs, based on the predicted response shown in $\boldsymbol{C}$. Blue and red lines depict the parameters calculated based on a Gabor RF model with $\theta=0$ and $\theta=\pi / 2$, respectively. Shown are the $\Delta F / F$ to the Pref, the $\Delta F / F$ to the Orth, OSI, 1 - CirVar, HWHH, and the preferred orientation.

in OSI ( $p<0.01$, Wilcoxon signed-rank test), a decrease in $1-\operatorname{CirVar}\left(p<10^{-4}\right.$, Wilcoxon signed-rank test), and a slight decrease in HWHH $(p<0.05$, Wilcoxon signedrank test). Here too, we found cells with a significant shift in their preferred orientation (Fig. 2A, right), and, indeed, the $\Delta$ in preferred orientation was $18.5 \pm 2.5^{\circ}$ (mean \pm SEM; Fig. 2C, right). Further analysis of the change in the preferred orientation in nonoptimal SFs revealed a monotonic averaged shift from SF below the preferred orientation to SF above the preferred orientation, which was quantified as the averaged shift across cells shown in Figure 2, C and D, and exemplified in the tuning curves of single cells shown in Figure 1D. The population results shown in Figure 2, A and B, were consistent across animals. The preferred response was decreased, as expected, at lower and higher SFs $(\Delta 4.73 \pm 0.74 \%$ and $\Delta 5.39 \pm 0.66 \%$, respectively; $n=5$ animals; $85 \pm 3.6$ cells; $t$ test, $p<0.005$ ); the Orth response was increased and decreased at lower and higher SFs, respectively
$(\Delta 0.94 \pm 0.31 \%$ and $\Delta 1.32 \pm 0.67 \%$, respectively; $t$ test, $p<0.05$ ); the mean OSI was significantly decreased at lower and higher SFs $(\Delta 0.26 \pm 0.05 \%$ and $\Delta 0.12 \pm$ $0.02 \%$, respectively; $t$ test, $p<0.01$ ); the mean (1 CirVar) was significantly decreased at lower and higher SFs $(\Delta 0.084 \pm 0.010 \%$ and $\Delta 0.087 \pm 0.009 \%$, respectively; $t$ test, $p<0.01$ ); the HWHH was increased and decreased at lower and higher SFs, respectively $(\Delta 6.7 \pm$ $1.7 \%$ and $\Delta 3.1 \pm 1.1 \%$, respectively; $t$ test, $p<0.05$ ), and the preferred orientation had a mean shift of $22.1 \pm 0.9^{\circ}$ at a lower SF and a $18.3 \pm 0.6^{\circ}$ shift at a higher SF (mean \pm SEM; Fig. 2E).

\section{A tilted Gabor model explains a shift in the preferred orientation at different SFs}

To our knowledge, the observed shift in the preferred orientation as a function of SF has not been previously reported in mice, although it has been demonstrated in cats (Jones et al., 1987). Therefore, we further investi- 
A
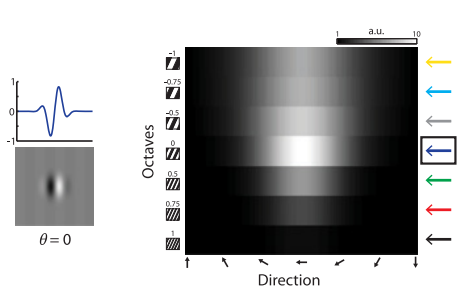

Altering the Gabor phase
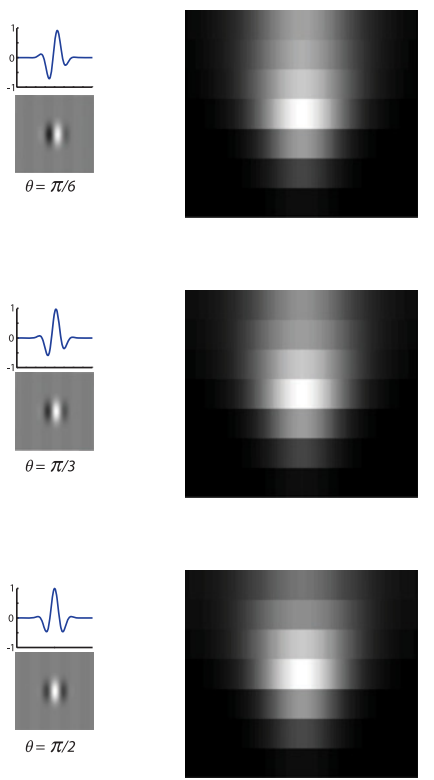
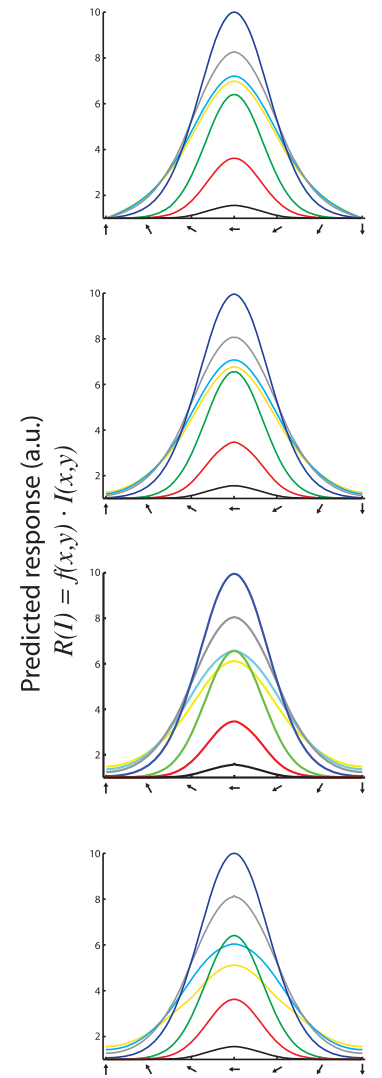

B
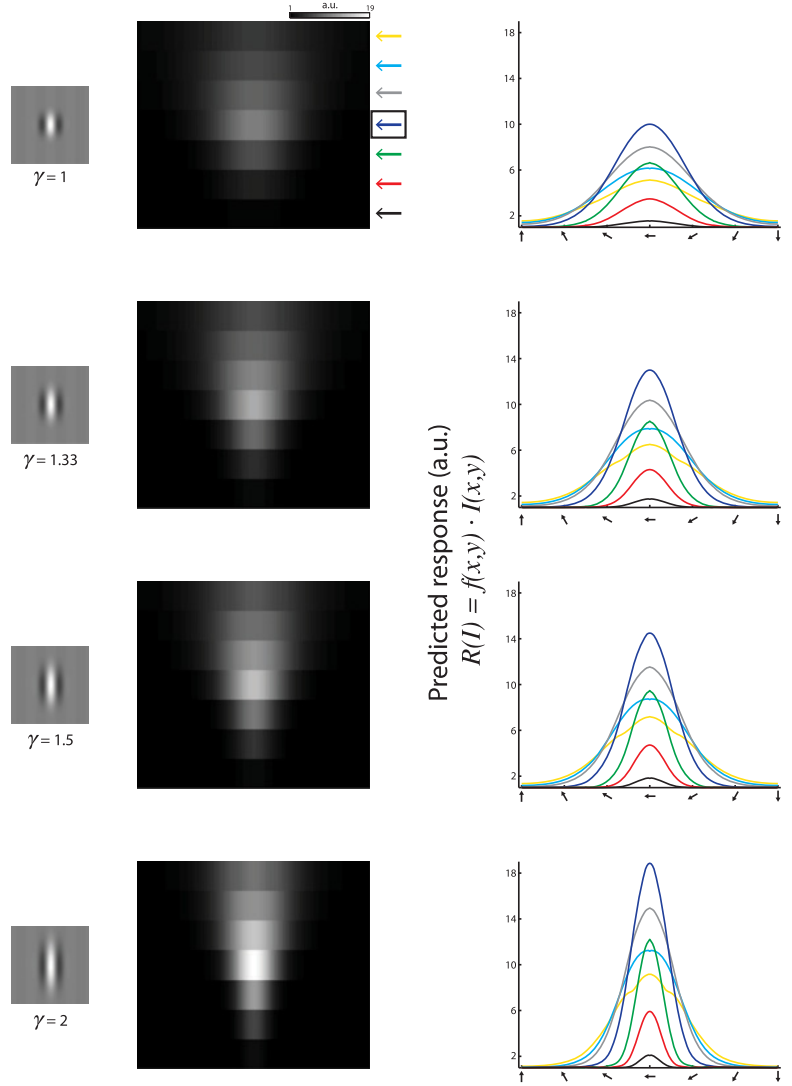

Figure 4. Altering the Gabor model parameters for predicting neural responses. A, A predicted response tuning matrix computed as the inner product between the Gabor RF model shown in Figure 3 and stimuli of square-wave gratings with various orientations ( $1^{\circ}$ interval) and seven SFs. Each pixel represents the inner product between the RF and the stimulus (maximized across phase). The row marked with a blue arrow on the right denotes the preferred SF and was taken as a reference for comparing other SFs. Right, Orientation-tuning curves at various SFs, color coded according to the arrows shown next to the predicted tuning matrix. Each panel corresponds to RF with $\theta=0, \theta=\pi / 6, \theta=\pi / 3$, and $\theta=\pi / 2$. a.u., Arbitrary units. $\boldsymbol{B}$, Same as in $\boldsymbol{A}$ only for an RF with $\theta=\pi / 2$ and various spatial aspect ratios $(\gamma)$. Each panel corresponds to RF with $\gamma=1, \gamma=1.33, \gamma=1.5$, and $\gamma=2$. Note that these parameter alterations alone could not qualitatively explain a shift in the preferred orientation of the cells at different SFs.

gated whether a known RF model of simple cells in V1 may explain this finding. To do so, we used a Gabor filter model with odd or even symmetry $(\theta=0$, or $\theta=\pi / 2$ respectively, where $\theta$ denotes the sine-wave phase; see Materials and Methods; Fig. 3A) to compute the predicted response of a neuron. A Gabor filter with odd symmetry consists of two side-by-side antagonistic regions of equal strength, whereas a Gabor filter with even symmetry demonstrates a central region flanked by two antagonistic regions of equal strength (Fig. 4A). The neuronal response is predicted based on the dot product between the Gabor filter and the stimulus (square-wave drifting gratings). Since we imaged $\mathrm{Ca}^{2+}$ signals that have slow dynamics, we could not deduce the optimal phase that each neuron preferred. Therefore, as the experimental tuning curves were calculated based on the maximum evoked responses, we used in our predictions the maximal response across phase (Fig. 5).

We calculated the predicted response for stimuli with various orientations and SFs, and, accordingly, computed the orientation-tuning curves at different SFs (Fig. 3C) and examined the behavior of their parameters (Fig. 3D). First, we observed a significant difference between a Gabor filter with odd symmetry and a Gabor filter with even symmetry in the predicted responses to the orthogonalto-preferred orientation. This difference also led to a difference in OSI by its definition and in 1 - CirVar. Second, and more importantly, we noticed that a symmetric Gabor filter, regardless of its phase, could not explain a shift in the preferred orientation of a neuron with a change in SF (Fig. 3D). Therefore, we also simulated the predicted response by altering different symmetry components of the Gabor filter [e.g., the phase of the sine wave $(\theta)$ and the spatial aspect ratio of the Gaussian envelope; $\gamma$; see Materials and Methods; also see Fig. 4A,B], but these alterations alone could not qualitatively explain a shift in the preferred orientation of the cells.

Since the classic Gabor model did not predict our experimental findings, we sought to examine alternative models. We found that one simple modification to the 

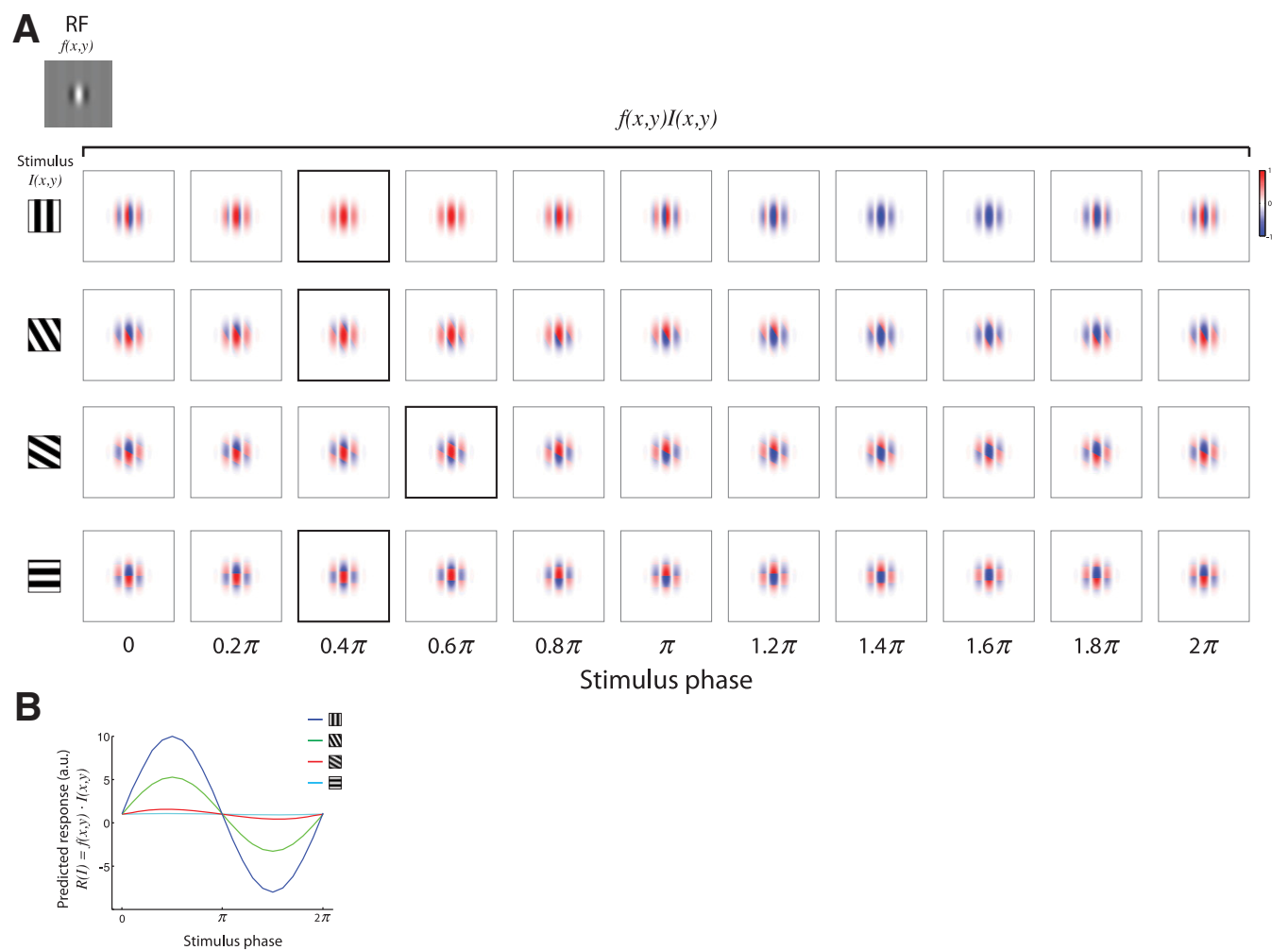

Figure 5. Impulse responses from gratings stimuli drifting against a Gabor RF. $\boldsymbol{A}$, The impulse responses from gratings stimuli drifting against a Gabor RF in various offsets. Top left, A Gabor RF with $\theta=\pi / 2$. In each row, on the left is the stimulus presented with one orientation; on the right are 11 examples where each box depicts the overlap of the grating stimulus crossing the RF in a particular phase. The box marked with a black border is the phase yielding the maximum response. $\boldsymbol{B}$, Bottom, The predicted response as a function of phase, calculated as the inner product between the RF and the stimulus at each phase. The predicted response per stimulus was then maximized across phase.

traditional model could explain the unexpected dependence of orientation on SF. We introduced an asymmetry by way of tilting the Gaussian envelope along the elongated axis of the 2D sine wave (Fig. 6A) and generated a filter that demonstrates a displacement of one subfield relative to the other along the RF orientation axis. We refer to this model as a tilted Gabor. Again, we generated two types of tilted Gabors according to the phase of the sine wave $(\theta=0$, or $\theta=\pi / 2)$ and then predicted the neuronal responses accordingly (Fig. 6B). We examined the dependence of orientation-tuning curves on SF (Fig. 6C) and found that using this modified RF we can predict the behavior of all parameters: a change in the Orth response, a change in OSI, a change in 1 - CirVar, a change in $\mathrm{HWHH}$, and most importantly, a shift in the preferred orientation of the neuron. Here too we observed a difference in the preferred orientation shift between RF phases, such that RF with $\theta=0$ showed a monotonic decrease with the increase in SF, whereas a tilted Gabor with $\theta=$ $\pi / 2$ showed a nonmonotonic behavior (Fig. 6D).

Collectively, the modified tilted Gabor model better explains our experimental data and suggests an asymmetric 2D organization of the RF ON and OFF subfields.

\section{Discussion}

In this study, we measured neuronal responses from layer $2 / 3$ of primary visual cortex of mice presented with drifting gratings of various orientations and SFs. We observed a unique dependence of orientation-tuning curves on SF, which suggests that the receptive field of some cells in mouse V1 present spatial asymmetry in their RF structural organization.

\section{Investigating tuning curves in populations of neurons using calcium imaging}

Both optical and electrical recording techniques enable the monitoring of activity from hundreds of neurons simultaneously. Therefore, to conduct feasible experiments, one cannot tailor the repertoire of stimuli to the optimal stimulus of each and every neuron. Therefore, the number and the complexity of the experimental stimuli are reduced. In the visual cortex, one common way to reduce the stimulus dimensionality is to measure 1D tuning curves, which are composed of neuronal responses evoked by presenting drifting gratings of various orientations (typically, 8 or 12). This is based on the assumption that the 2D spatial structure of the receptive field can account for a large fraction of the orientation selectivity of simple cells (Lampl et al., 2001). However, orientationtuning curves measured with a small number of points are just a 1D reduction of the receptive field of a neuron, which has a more complex structure in a highdimensional space. As a result of this dimensionality reduction, we not only "pay" a quantitative price of reduced 

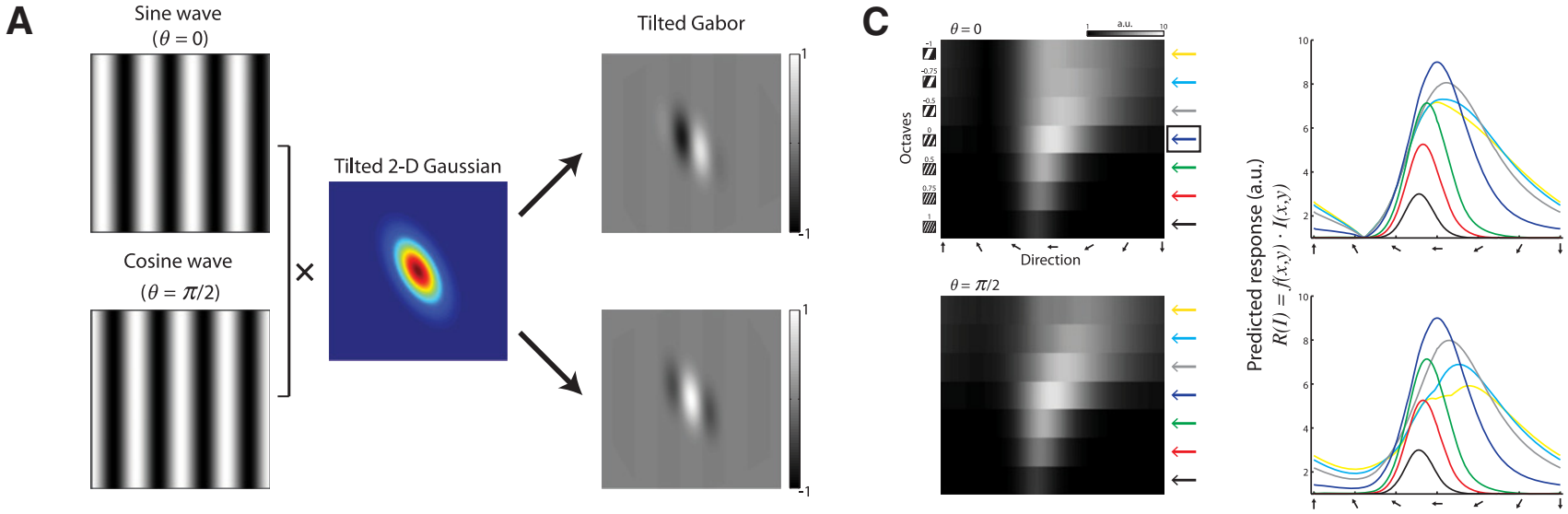

B

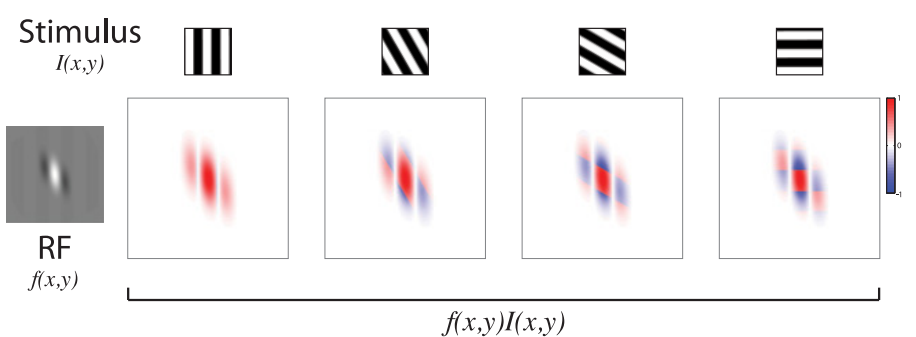

D
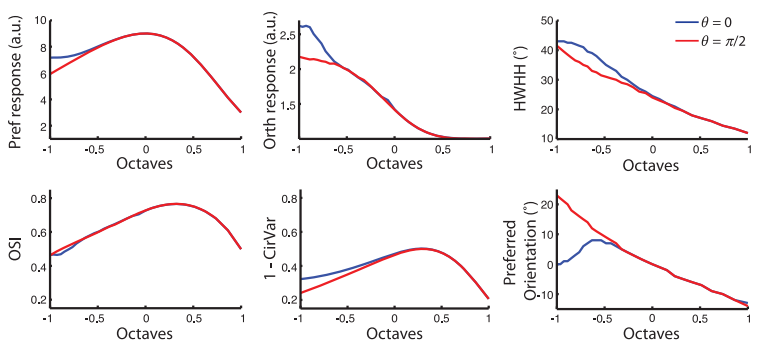

Figure 6. Predicting the responses of a simple cell based on an RF model of a 2D tilted Gabor. A, A 2D tilted Gabor function: a sinusoidal plane wave weighted by a tilted Gaussian envelope (the Gaussian was rotated against the orientation of the sinusoidal plane wave) in two different phases: $\theta=0$ (sine wave) or $\theta=\pi / 2$ (cosine wave) shown at the top and bottom panels, respectively. $\boldsymbol{B}$, Constructing spatiotemporally oriented impulse responses from gratings stimuli drifting against a tilted Gabor RF. Top, Examples of stimuli with four various orientations. Bottom, Left shows an example of a tilted Gabor RF with $\theta=\pi / 2$. On the right, four examples where each box depicts the overlap of the grating stimulus crossing the RF in a phase that yields the maximum response, calculated as the inner product between the stimulus and the RF. C, A predicted response tuning matrix computed as the inner product between the tilted Gabor RF model shown in $\boldsymbol{A}$, and stimuli of square-wave gratings with various orientations ( $1^{\circ}$ interval) and seven SFs. Each pixel represents the inner product between the RF and the stimulus (maximized across phase). The row marked with a blue arrow on the right denotes the preferred SF and was taken as a reference for comparing other SFs. Right, Orientation tuning curves at various SFs, color coded according to the arrows shown next to the predicted tuning matrix. Top and bottom panels correspond to RF with $\theta=0$ and $\theta=\pi / 2$, respectively. $\boldsymbol{D}$, Comparison of orientation-tuning parameters between various SFs, based on the predicted response shown in $\boldsymbol{C}$. Blue and red lines depict the parameters calculated based on a tilted Gabor RF model with $\theta=0$ and $\theta=\pi / 2$, respectively. Shown are the $\Delta F / F$ to the Pref, the $\Delta F / F$ to the Orth, OSI, $1-$ CirVar, HWHH, and the preferred orientation.

neural responses due to nonoptimal stimulus, but we also pay a qualitative price, manifested as a shift in the preferred orientation of the cells in nonoptimal SFs that likely arises from an asymmetric RF structure.

\section{Cortical neurons have an asymmetric RF spatial structure}

The RF structure of a neuron in visual cortex describes the organization of ON and OFF subfields in visual space, and accordingly explains which visual features a neuron is sensitive to. Simple cells in primate and cat cortex have been found to present mainly 1D symmetric organization (i.e., odd-phase or even-phase symmetry along the $x$-axis, orthogonal to the preferred orientation), although there are studies showing that cells do not demonstrate only odd or even symmetry but also exhibit, to a smaller extent, various phases, which breaks the 1D symmetry that exists between the relative strength of each subfield (Kulikowski and Bishop, 1981; Jones and Palmer, 1987a; Ringach, 2002).
However, breaking the amplitude symmetry alone cannot explain the behavior of the observed tuning parameters (Fig. 4). Only a 2D spatial asymmetry that introduces a shift in the relative location of the subfields can predict our experimental findings. In fact, the critical characteristic in predicting a shift in the preferred orientation is the displacement of one RF-subfield relative to the other along the orientation axis, such that it breaks the symmetry in the orthogonal axis. This 2D displacement can be easily modeled by introducing a tilt in the 2D Gaussian envelope that generates an asymmetric Gabor filter.

Traditionally, the Gabor filter has been proposed as a model to describe the receptive field of simple cells, and successfully predicts the responses of cortical neurons of monkeys and cats (Marcelja, 1980; Kulikowski et al., 1982; Daugman, 1985; Field and Tolhurst, 1986; Jones and Palmer, 1987b). The Gabor model is optimal in terms of minimizing the uncertainty associated with localizing a 
signal in both space and SF (Marcelja, 1980; Daugman, 1985), and therefore can create a sparse representation of natural images. The modified model we present here keeps the basic characteristics of the classic model and introduces one additional parameter that accounts for spatial asymmetry.

Although the tilted model succeeds in predicting the observed responses, there is still no experimental study in mice that mapped the RF of cells directly, and accordingly characterized asymmetry based on observations. However, a study in cats (Jones and Palmer, 1987a) showed that the $2 \mathrm{D}$ response profile of simple cells is not necessarily Cartesian separable due to a relative displacement of the subfields, and that within a given receptive field, subfields need not be the same length. In addition, visual inspection of examples of cells from studies conducted in mice also revealed some displacement between the ON and OFF subfields of the RF of cells (Bonin et al., 2011; Ko et al., 2013; Cossell et al., 2015). However, Bonin et al. (2011) used wavelet stimuli to map the RF of cells (Selesnick et al., 2005), which are spatially symmetric by nature, while Ko et al. (2013) and Cossell et al. (2015) presented natural images but used a regularized pseudoinverse method to estimate the RF of cells (Smyth et al., 2003), which introduces a two-dimensional smoothness constraint on the RF and might bias the RF structure toward more symmetric organization.

\section{Functional role of asymmetry in neuronal representations}

Breaking the symmetry of the receptive field of neurons has been previously reported in the retina and the hippocampus. In the retina, asymmetry is observed between the sizes of the circular receptive fields of ON and OFF ganglion cells (Chichilnisky and Kalmar, 2002), or between the relative dendritic field size of parasol and midget cells (Dacey and Petersen, 1992), or in the asymmetric adaptation of ON and OFF ganglion cells to photopic (day) and scotopic (night) conditions (Pandarinath et al., 2010).

Hippocampal place cells exhibit an asymmetry in the spatial arrangement of their place fields. That is, as the animal moves through the receptive field of a cell, the firing rate modulates asymmetrically, where at the start of the place field, the firing rate is low, and at the end of the field, the firing rate is higher. The asymmetry increases as a function of familiarity (Mehta et al., 2000). This asymmetry also exists at the level of subthreshold excitatory inputs to place cells, and has been proposed to arise from a change in the balance between inhibitory inputs at the pyramidal cell soma and increases in dendritic excitation (Harvey et al., 2009). This place cell asymmetry represents a prospective coding scheme, where the increased firing skewness early in the receptive field signals upcoming place field center. The network mechanisms of this asymmetry however, are not completely known.

Furthermore, asymmetry in the visual cortex might play an important role in contour integration, which is in line with the gestalt principles of perceptual grouping (Metzger, 2006), specifically the law of "good continuation" (Field et al., 1993). Since neighboring neurons share
RF subfields (Smith and Häusser, 2010), then the integration of local populations of neurons, some of which exhibit spatial RF asymmetry, may be the basis for cortical representation of spatial continuity in curved shapes.

\section{References}

Andrews BW, Pollen DA (1979) Relationship between spatial frequency selectivity and receptive field profile of simple cells. $J$ Physiol 287:163-176. Medline

Bonin V, Histed MH, Yurgenson S, Reid RC (2011) Local diversity and fine-scale organization of receptive fields in mouse visual cortex. J Neurosci 31:18506-18521. CrossRef Medline

Brainard DH (1997) The Psychophysics toolbox. Spat Vis 10:433436. Medline

Chichilnisky EJ, Kalmar RS (2002) Functional asymmetries in ON and OFF ganglion cells of primate retina. J Neurosci 22:2737-2747. Medline

Cossell L, lacaruso MF, Muir DR, Houlton R, Sader EN, Ko H, Hofer SB, Mrsic-Flogel TD (2015) Functional organization of excitatory synaptic strength in primary visual cortex. Nature 518:399-403. CrossRef Medline

Dacey DM, Petersen MR (1992) Dendritic field size and morphology of midget and parasol ganglion cells of the human retina. Proc Natl Acad Sci U S A 89:9666-9670. Medline

Daugman JG (1985) Uncertainty relation for resolution in space, spatial frequency, and orientation optimized by two-dimensional visual cortical filters. J Opt Soc Am A 2:1160-1169. Medline

Field DJ, Tolhurst DJ (1986) The structure and symmetry of simplecell receptive-field profiles in the cat's visual cortex. Proc R Soc Lond B Biol Sci 228:379-400. Medline

Field DJ, Hayes A, Hess RF (1993) Contour integration by the human visual system: evidence for a local "association field". Vision Res 33:173-193. Medline

Gabor D (1946) Theory of communication. J IEE 93:429-457. CrossRef

Harvey CD, Collman F, Dombeck DA, Tank DW (2009) Intracellular dynamics of hippocampal place cells during virtual navigation. Nature 461:941-946. CrossRef Medline

Hubel DH, Wiesel TN (1962) Receptive fields, binocular interaction and functional architecture in the cat's visual cortex. J Physiol 160:106-154. Medline

Jones JP, Palmer LA (1987a) The two-dimensional spatial structure of simple receptive fields in cat striate cortex. J Neurophysiol 58:1187-1211.

Jones JP, Palmer LA (1987b) An evaluation of the two-dimensional Gabor filter model of simple receptive fields in cat striate cortex. $J$ Neurophysiol 58:1233-1258. Medline

Jones JP, Stepnoski A, Palmer LA (1987) The two-dimensional spectral structure of simple receptive fields in cat striate cortex. J Neurophysiol 58:1212-1232. Medline

Kerlin AM, Andermann ML, Berezoversuskii VK, Reid RC (2010) Broadly tuned response properties of diverse inhibitory neuron subtypes in mouse visual cortex. Neuron 67:858-871. CrossRef Medline

Kleiner M, Brainard D, Pelli D (2007) What's new in Psychtoolbox-3? Perception. Paper presented at the Thirtieth European Conference on Visual Perception, Arezzo, Italy, August.

Ko H, Cossell L, Baragli C, Antolik J, Clopath C, Hofer SB, MrsicFlogel TD (2013) The emergence of functional microcircuits in visual cortex. Nature 496:96-100. CrossRef Medline

Kulikowski JJ, Bishop PO (1981) Linear analysis of the responses of simple cells in the cat visual cortex. Exp Brain Res 44:386-400. Medline

Kulikowski JJ, Marcelja S, Bishop PO (1982) Theory of spatial position and spatial frequency relations in the receptive fields of simple cells in the visual cortex. Biol Cybern 43:187-198. Medline

Lampl I, Anderson JS, Gillespie DC, Ferster D (2001) Prediction of orientation selectivity from receptive field architecture in simple cells of cat visual cortex. Neuron 30:263-274. Medline 
Marcelja S (1980) Mathematical description of the responses of simple cortical cells. J Opt Soc Am 70:1297-1300. Medline

Marshel JH, Garrett ME, Nauhaus I, Callaway EM (2011) Functional specialization of seven mouse visual cortical areas. Neuron 72: 1040-1054. CrossRef Medline

Mazurek M, Kager M, Van Hooser SD (2014) Robust quantification of orientation selectivity and direction selectivity. Front Neural Circuits 8:92. CrossRef Medline

Mehta MR, Quirk MC, Wilson MA (2000) Experience-dependent asymmetric shape of hippocampal receptive fields. Neuron 25: 707-715. Medline

Metzger W (2006) Laws of seeing. Cambridge, MA: MIT.

Miller JE, Ayzenshtat I, Carrillo-Reid L, Yuste R (2014) Visual stimuli recruit intrinsically generated cortical ensembles. Proc Natl Acad Sci U S A 111:E4053-E4061. CrossRef Medline

Niell CM, Stryker MP (2008) Highly selective receptive fields in mouse visual cortex. J Neurosci 28:7520-7536. CrossRef Medline

Nimmerjahn A, Kirchhoff F, Kerr JN, Helmchen F (2004) Sulforhodamine 101 as a specific marker of astroglia in the neocortex in vivo. Nat Methods 1:31-37. CrossRef Medline

Pandarinath C, Victor JD, Nirenberg S (2010) Symmetry breakdown in the ON and OFF pathways of the retina at night: functional implications. J Neurosci 30:10006-10014. CrossRef Medline

Pelli DG (1997) The VideoToolbox software for visual psychophysics: transforming numbers into movies. Spat Vis 10:437-442. Medline

Piscopo DM, El-Danaf RN, Huberman AD, Niell CM (2013) Diverse visual features encoded in mouse lateral geniculate nucleus. $J$ Neurosci 33:4642-4656. CrossRef Medline

Ringach DL (2002) Spatial structure and symmetry of simple-cell receptive fields in macaque primary visual cortex. J Neurophysiol 88:455-463. Medline
Ringach DL, Shapley RM, Hawken MJ (2002) Orientation selectivity in macaque V1: diversity and laminar dependence. J Neurosci 22:5639-5651. Medline

Selesnick IW, Baraniuk RG, Kingsbury NC (2005) The dual-tree complex wavelet transform. IEEE Signal Process Mag 22:123-151. CrossRef

Skottun BC, De Valois RL, Grosof DH, Moversushon JA, Albrecht DG, Bonds AB (1991) Classifying simple and complex cells on the basis of response modulation. Vision Res 31:1079-1086. Medline

Smith SL, Häusser M (2010) Parallel processing of visual space by neighboring neurons in mouse visual cortex. Nat Neurosci 13: 1144-1149. CrossRef Medline

Smyth D, Willmore B, Baker GE, Thompson ID, Tolhurst DJ (2003) The receptive-field organization of simple cells in primary visual cortex of ferrets under natural scene stimulation. J Neurosci 23: 4746-4759. Medline

Thévenaz P, Ruttimann UE, Unser M (1998) A pyramid approach to subpixel registration based on intensity. IEEE Trans Image Process 7:27-41. CrossRef Medline

Vidyasagar TR, Sigüenza JA (1985) Relationship between orientation tuning and spatial frequency in neurones of cat area 17. Exp Brain Res 57:628-631. Medline

Webster MA, De Valois RL (1985) Relationship between spatialfrequency and orientation tuning of striate-cortex cells. J Opt Soc Am A 2:1124-1132. Medline

Zhu W, Xing D, Shelley M, Shapley R (2010) Correlation between spatial frequency and orientation selectivity in V1 cortex: implications of a network model. Vision Res 50:2261-2273. CrossRef Medline 